Methods: 30 undergraduate medical students (January 2017) were given modified teaching material for a 60 minute teaching session a week later. This material involved a partially populated Microsoft Powerpoint slideshow including prompts about a theoretical patient's clinical case in the notes section. This guided development of a clinical case presentation which covered diverse aspects of clinical care, e.g. imaging, extra-articular disease, drug side effects and disease activity scores. Clinical cases addressed systemic lupus erythematous, early rheumatoid arthritis (RA), ankylosing spondylitis and established RA. Students (groups of 2-3) received one case each were encouraged to use images and online teaching repositories to enhance their presentation. This provided the framework for a 20 minute teaching presentation which was given to their student peers at a formal teaching day 1 week later. A tutor was also present during these sessions (with a ratio of 1:10) to ensure adequate understanding of topics had been achieved and to answer any questions. Feedback was sought from the students and compared with previous "paper case" (non-modified) sessions.

Results: Feedback obtained $(n=9)$ showed $55 \%$ of students rated the modified teaching session as "excellent" with the remainder rating it as "good". Free-text comments included "good to have students to do the presentations so they cover relevant points" "very useful to have a quick 20 minute overview of different conditions \& preparation was useful" and "lots of learning, interactive". Additional comments included the wish for more time to cover the points in even more depth. Informal feedback from the tutors of these events was also favourable with tutors believing students had a developed a greater depth of understanding. These findings compared favourably with the previous years "paper-case" feedback (March 2016, $n=13)$ where only $23 \%(n=3)$ of students had rated the session as "excellent" as well as free text comments emphasising wishes for more time to read through cases and smaller group discussion.

Conclusions: Current "paper based" modalities can easily be utilized and "re-purposed" to optimize both self-directed and formal teaching components of undergraduate teaching. This can promote the understanding of complex rheumatological learning points in a relatively short period of time and allow students exposure to modalities, such as imaging, which may previously be excluded in a traditional "paper case" format.

Disclosure of Interest: None declared

DOI: 10.1136/annrheumdis-2017-eular.6451

\section{AB1187 SURVEY ON GENERIC DRUGS (GE) AND BIO-SIMILAR DRUGS (BIO-S) OF PATIENTS WITH RHEUMATOID ARTHRITIS (RA) AND THEIR DOCTORS - COHORT STUDY OF THE JAPANESE CLINICIAN BIOLOGICS RESEARCH GROUP}

K. Funahashi ${ }^{1}$, T. Yoshitama ${ }^{2}$, T. Tetsu Oyama ${ }^{3}$, A. Sagawa ${ }^{3}$, K. Katayama $^{3}$, T. Matsubara $^{3}$, N. Nishimoto ${ }^{3}$, S. Kiyokawa ${ }^{3}$, N. Miyake ${ }^{3}$, A. Akihiko Nakamura $^{3}$, M. Oribe ${ }^{3}$, T. Tomohiko Yoshida ${ }^{3}$, T. Mitsuka ${ }^{3}$ on behalf of Japanese Clinician Biologics Research Group. ${ }^{1}$ Clinical Research, Matsubara

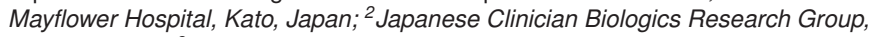
Kobe, Andorra; ' Japanese Clinician Biologics Research Group, Kobe, Japan

Background: In Japan in 2014 the market share for generic drugs was $50 \%$, lower than that of Western nations, but the reasons why the patients themselves turn down the opportunity to switch to a generic drug when asked at the pharmacy are not clear. In addition, the use of bio-similar drugs became possible in Japan in 2015, but the degree of knowledge that patients have regarding them is unknown. Objectives: Therefore we carried out a patient survey about generic and biosimilar drugs, and at the same time, we also conducted a questionnaire for doctors who prescribed them, and conducted a comparative study of patient's and doctor's awareness regarding these drugs.

Methods: The survey was carried out amongst 4151 patients being treated at 20 research group member facilities. It was an anonymous written survey. After the section on patient background (age, gender, disease history) was completed, patients were asked their impressions of generic drugs, their attitudes towards changing to a generic, whether or not they had ever experienced an adverse effect with a generic drug, what knowledge they had regarding bio-similar drugs, and if they had any interest in or experience with using bio-similar drugs.We also asked 32 physicians about permissions and restrictions on GE use, negative experience of GE, problems of GE, experience of BIO-S and future plans for use, conditions of $\mathrm{BIO}-\mathrm{S}$ usage, etc.

Results: The group was $78 \%$ female, the majority of whom were in their 60 's, and most had disease history of more than 10 years. Those with a good impression of GE drugs comprised $41 \%$, those with a bad impression $9.4 \%$. 34\% of patients had switched to GE drugs, those who would not or could not were $24 \%$. Those who reported a bad experience (lessening of effectiveness, or an adverse effect) were $8 \%$.Meanwhile, in GE survey of doctors, about $65 \%$ partially restricted the use of GE, and about $70 \%$ had experiences of weakening of effects and occurrence of adverse events when using GE. Regarding patient knowledge of BIO-S, $13 \%$ knew of them, and $44 \%$ replied that they had no interest in them, even after being shown explanatory materials about the drugs. $63 \%$ of patients said they would rely on their physicians's judgement regarding choosing BIO-S drugs, with $10 \%$ stating they would choose BIO-S based on cost benefits. On the other hand, approximately $40 \%$ of physicians have experience using BIO - S, while about $60 \%$ have experience explaining them to patients. About $60 \%$ replied that they plan to use BIO-Ss to be released in the future but on the condition of regular and detailed provision of safety information.
Conclusions: Due to experience in using GE, physicians wish to receive detailed information on individual drugs, and desire it even more in the case of BIO-S drugs. RA patients with RA have received little information on BIO-S, and this can be thought to be a direct result of lack of information for prescribing physicians. Disclosure of Interest: None declared

DOI: 10.1136/annrheumdis-2017-eular.2288

\section{AB1188 WHAT IS THE VALUE OF RHEUMATOLOGY ON-CALL SERVICE? AN EVALUATION OF ACUTE RHEUMATOLOGY ON-CALL SERVICE IN A TERTIARY CENTRE}

\section{L.H. Lee, A. Gupta. Nottingham University Hospitals NHS Trust, Nottingham,} United Kingdom

Background: Rheumatology is largely an outpatient specialty. Changes in healthcare structure in recent years have seen a trend for increased demand for specialty input in acute inpatient care. However, financial pressures on the other hand have led to calls to curtail specialty on-call service. Service provision apart, "being on-call" has traditionally been seen as an important educational experience, equipping registrars with valuable acute rheumatology skills. Despite all these, there is little published literature evaluating the contribution and workload of Rheumatology on-call service.

Objectives: This study sought to evaluate the contribution of Rheumatology on-call service as well as to assess the spectrum of conditions referred.

Methods: An electronic database was created to record details of on-call referrals received by one Rheumatology specialty registrar in a tertiary hospital. These included relevant clinical details, outcomes of referral and working diagnosis.

Results: A total of 40 referrals were recorded over 9 days in a 5-week period from December 2016 to January 2017. Seventeen referrals were reviewed on the same day by the rheumatology registrar. Referrals from medical wards comprised 10 out of 17 referrals reviewed. Other sources of referrals were Emergency Department, General Practitioners and Rheumatology advice phone line with 4, 2 and 1 referrals respectively.

Of the 17 patients reviewed on-call, 11 referrals $(65 \%)$ could be classified in the category of "acute hot joint", 2 were in the category of vasculitis and connective tissue disorders, 2 were predominantly mechanical problems, 1 was referred with a painful limb while 1 had an elevated creatine kinase. Thirteen of these 17 patients required an arthrocentesis, 12 of which were either performed or supervised by the rheumatology team. Septic arthritis was diagnosed in only one patient. Three of the 17 patients had musculoskeletal ultrasound scan performed by the rheumatology team on the same day.

Conclusions: Rheumatology on-call service provides a significant contribution to acute care which cannot be substituted by other specialties. It also continues to provide rheumatology trainees with experience in acute rheumatology although it could be argued that the spread of conditions seen were skewed toward acute hot joints and that exposure to connective tissue disorders and vasculitis was limited. The results of this study have important implications for planning and delivery of postgraduate Rheumatology training curriculum and delivery of on-call Rheumatology service.

Disclosure of Interest: None declared

DOI: 10.1136/annrheumdis-2017-eular.5537

\section{AB1189 "GO TRANS": OUTCOME OF THE PROGRAM OF WORKSHOPS} FOR ADOLESCENTS WITH RHEUMATOLOGICAL DISEASES

M.A. Blázquez ${ }^{1}$, A. Boteanu ${ }^{1}$, L. León ${ }^{2}$, S. Lerma ${ }^{3}$, M. Vázquez ${ }^{1} .{ }^{1}$ Hospital $^{2}$ Universitario Ramón y Cajal; 22 Idissc, Hospital Universitario Clínico San Carlos Madrid; ${ }^{3}$ Hospital Niño Jesús, Madrid, Spain

Background: Transition is the process by which a young patient with a chronic disease is able to develop skills and have access to resources to ensure that physical, psychological, educational and vocational needs are covered during the stage from youth to adulthood. According to SERPE and EULAR/PReS recommendations, the goal of transition programs is to improve the support to patients during this process, mainly working in multidisciplinary teams.

Objectives: Our main purpose was to accomplish self-care, illness awareness, stress management and negative emotions workshops focused in improving their life quality.

Methods: Workshops are conducted by a psychologist, a pediatric physiotherapist, two rheumatologists and a rheumatology nurse at our center. It consists of a total of 7 workshops (first six for patients and the last for parents). Five of these workshops are based on HEADSS system (home, education, activities, drugs, sexual activity, and emotions) and the two others are focused on disease and treatment consciousness. Parents signed an informed consent document. Workshops lasted 90 minutes in sessions outside of the consultation hours. At baseline, several questionnaires were completed, VAS pain (parents and patients), VAS general (parents and patients), JAMAR (parents and patients), PedsQL 4.0, those questionnaires were also completed after 3 months together with a satisfaction survey.

Results: A total of 12 patients were included, with ages between 11 to 16 years old, with an average of 14 years old. A total of 10 patients completed $100 \%$ of the program ( 6 girls and 4 boys). 6 patients had AlJ (oligoarticular, enthesitis-related arthritis, psoriatic arthritis), 3 suffered from LES and 1 had Behçet. All patients 